\title{
HIF, hypoxia and the role of angiogenesis in non-small cell lung cancer
}

\author{
Autumn L Jackson, Bing Zhou, and William Y Kim ${ }^{\dagger}$ \\ The University of North Carolina, The Lineberger Comprehensive Cancer Center, Chapel Hill, \\ NC, USA
}

\begin{abstract}
Importance of the field-The role of angiogenesis in the initiation and progression of NSCLC and the molecular alterations leading to the growth of tumor vasculature are areas of great interest and recent therapeutic success.
\end{abstract}

\begin{abstract}
Areas covered in this review-VEGF and its receptors play critical roles in the development of tumor vasculature and can be targeted by agents such as bevacizumab in the treatment of NSCLC. Furthermore, tumor hypoxia and the expression of the hypoxia-inducible factor (HIF) family of proteins are also linked to poorer survival in these patients. Recent studies using genetically engineered mouse models expressing stabilized HIF validate the importance of HIF in the evolution of NSCLC and demonstrate genetically that HIF is involved in NSCLC.
\end{abstract}

What the reader will gain-An overview of the key pathways and mediators of tumor angiogenesis, their relevance to the pathogenesis of NSCLC, and an update on the current status of angiogenesis inhibitors in NSCLC.

Take home message-Angiogenesis is a key mediator of NSCLC progression. Several antiangiogenic strategies are in clinical use and under development. While candidate predictive biomarkers of response to antiangiogenic therapy exist, they await independent and prospective validation.

\section{Keywords}

angiogenesis; bevacizumab; hypoxia; hypoxia-inducible factor; lung cancer; sorafenib; sunitinib; vandetinib; vascular endothelial growth factor

\section{Introduction}

Lung cancer is the leading cause of cancer-related deaths in both men and women. In 2009, it is estimated that approximately 160,000 people will die of lung cancer in the USA, which is more than colon, breast, and prostate cancer deaths combined [1]. Non-small cell lung cancer (NSCLC) represents $80-85 \%$ of lung cancer cases and $70 \%$ of patients present with advanced-stage disease. With a response rate of only $20 \%$ to combination chemotherapy, the median survival of patients presenting with advanced disease is a mere 10 months [2]. Therefore, novel approaches to treat such a common killer are urgently needed.

(C) 2010 Informa UK, Ltd.

${ }^{\dagger}$ Author for correspondence The University of North Carolina, The Lineberger Comprehensive Cancer Center, CB\# 7295, Chapel Hill, NC 27599-7295, USA Tel: +1 919966 4765; Fax: +1 919966 8212; wykim@ med.unc.edu.

Declaration of interest The authors declare no conflict of interest. 
The role of angiogenesis in the initiation and progression of NSCLC and the molecular pathways leading to the growth of tumor vasculature have emerged as areas of great interest and therapeutic success in translational research. The VEGF and its receptors, VEGFR1, VEGFR2 and VEGFR3, are now well documented to play critical roles in the development of tumor vasculature and can be targeted by agents such as bevacizumab in the treatment of NSCLC [3]. Tumor hypoxia and the expression of the hypoxia-inducible factor (HIF) family of proteins are also linked to poorer survival in these patients. Therefore, more attention is being placed on the intricate relationship between hypoxia, angiogenesis and the development of NSCLC with an emphasis on targeting these processes to improve clinical outcomes.

\section{Key molecules involved in tumor angiogenesis}

Angiogenesis is defined as the growth of new vessels from an area of pre-existing vasculature. During the process of solid tumor formation and progression, tumors require an adequate supply of oxygen and nutrients. At early stages of growth, tumors often rely on vessels from the host vascular system. Ultimately, however, as tumors exceed a size that outgrows their vascular supply, they require the stimulation of neoangiogenesis (the sprouting of vessels from pre-existing capillaries) and/or vasculogenesis (the development of vessels primarily through the recruitment of bone-marrow-derived endothelial precursor cells) for their growth [4,5]. This step, termed the 'angiogenic switch', occurs when the balance of pro-angiogenic factors (e.g., VEGFA, placental growth factor (PIGF), IL-8, and angiopoietin-1 (ANG1)) outweighs anti-angiogenic factors (e.g., thrombospondin-1, angiostatin, etc.) [5-7].

Physiologic angiogenesis proceeds in a rapid and well-organized fashion resulting in mature and non-permeable vessels that are bolstered by supporting pericytes. These endothelial cell - pericyte interactions normally restrain endothelial cell proliferation and reduce their dependence on VEGF for survival [8]. In contrast, tumor vascular development is disorganized, resulting in blood vessels that are irregular in shape and size, tortuous and sometimes even incorporate cancer cells into the vessel wall (so called vasculogenic mimicry) [9-11]. These characteristics of tumor vessels along with an increased intratumoral pressure (secondary to increased vascular permeability) can create variable and turbulent blood flow, at times even resulting in the reversal of flow within a vessel [12]. Collectively, these abnormalities result in changes of the tumor microenvironment such as the development of hypoxia, acidosis and interstitial hypertension that may play a role in the ineffective delivery of therapeutics as well as the selection of more malignant cells with increased potential for metastases [9]. Whether or not the normalization of tumor vasculature results in the effective delivery of therapeutics or improves tumor oxygenation remains to be determined.

\subsection{The hypoxia-inducible factor (HIF)}

Intratumoral hypoxia results in the stabilization the HIF family of transcription factors. HIF is a heterodimeric transcription factor composed of a labile alpha subunit and a stable beta subunit [13]. The human genome contains three HIFa genes (HIF1a, HIF2a and HIF3a) and three HIF $\beta$ genes [13]. Under low oxygen conditions, HIFa accumulates, binds to HIF $\beta$, and transcriptionally activates genes whose promoters contain hypoxia-response elements (HREs), many of which are involved in the adaptation to both cellular and organismal hypoxia. These include genes involved in the uptake and metabolism of glucose (GLUT1, LDH), apoptosis (BNIP3), angiogenesis ( VEGFA, PDGF), control of extracellular $\mathrm{pH}(C A 9)$, mitogenesis ( $T G F a)$, and erythropoiesis (EPO) [13]. 
HIF is polyubiquitinated by the VHL (von Hippel-Lindau) gene product, pVHL. The binding of pVHL to HIF is governed by an oxygen- and iron-dependent, post-translational prolyl hydroxylation of HIFa subunits [14]. Thus, HIFa subunits are normally degraded in the presence of oxygen but are stabilized under hypoxic conditions or in the setting of pVHL loss, leading to transactivation of HIF target genes. While HIF protein levels are primarily determined by the pVHL status of cells as well as oxygenation levels, HIF mRNA and protein synthesis is largely regulated by oxygen-independent mechanisms, such as activation of the MAPK and PI3K pathways [15]. For example, HIF can be upregulated at the transcriptional level by activating mutations in $R A S$ family members or in $B R A F$ with resultant increases in HIF mRNA levels [16,17]. Additionally, translation of HIF mRNA is dependent upon mammalian target of rapamycin (mTOR) activity, as inhibition of the mTOR/Raptor complex by drugs such as rapamycin or rapamycin analogs result in lowered HIF protein levels [18-20]. This effect is at least partially mediated by the presence of terminal oligopyrimidine (TOP) tracts located in the $5^{\prime}$ untranslated region ( $5^{\prime}$ UTR) of both HIF1 and HIF2 as protein levels of HIF variants lacking their native $5^{\prime}$ UTRs are insensitive to mTOR inhibitors and their growth inhibitory effects [19].

Despite significant sequence homology, the functions of HIF1a and HIF2a appear to differ. While germline deletion of either gene in mice results in embryonic lethality, both the timing and cause of death differ. In HIF $1 a^{-/-}$mice, death occurs in mid-gestation with evidence of vascular defects [21]. In contrast, mice lacking HIF2a die earlier in embryogenesis with defects in lung development and catecholamine deficiency [22,23]. Finally, multiple studies have now shown that HIF1 and HIF2 activation produce overlapping yet distinct gene expression profiles and that certain target genes are uniquely regulated by either HIF1 or HIF2 both in vitro and in vivo [24-28].

\subsection{VEGF and VEGF receptors}

One of the key downstream targets of the HIF pathway is VEGF, a family of endothelial growth factors that plays a central role in angiogenesis through their effects on endothelial cell migration, proliferation, permeability and survival [29]. VEGF-A (henceforth referred to as VEGF) is the most abundant of the ligands and binds to VEGFR-1 and VEGFR-2 (also known as FLT1 and KDR, respectively), the latter being considered a critical receptor in regulating angiogenesis (Figure 1). Therefore, VEGFR-2 remains the primary target of most anti-angiogenic therapies. VEGFR-3 (also known as FLT4) is predominantly located on lymphatic endothelial cells and through binding with its ligands, VEGF-C and D, is thought to be primarily responsible for lymphagiogenesis [29].

Through alternative splicing of the full length VEGF mRNA, several isoforms of VEGF-A have been described, namely $\mathrm{VEGF}_{121}, \mathrm{VEGF}_{145}, \mathrm{VEGF}_{165}, \mathrm{VEGF}_{189}$, and $\mathrm{VEGF}_{206}$ [29]. These forms differ primarily on the presence or absence of heparin-binding domains encoded by exons 6 and 7, which decreases their bioavailability by tethering VEGF to the extracellular matrix $(\mathrm{ECM}) . \mathrm{VEGF}_{121}$ is freely diffusible as it is lacking this domain and is hence highly soluble. In contrast, $\mathrm{VEGF}_{189}$ and $\mathrm{VEGF}_{206}$ are highly basic and therefore bind heparin tightly, sequestering them on both the cell surface and the ECM (extracellular matrix). $\mathrm{VEGF}_{145}$ and $\mathrm{VEGF}_{165}$, the most abundant isoforms, have intermediate properties and are secreted as well as partially bound by the ECM. The sequestered isoforms can, however, be released by plasmin-mediated cleavage of the $\mathrm{COOH}$ terminus or by loss of the heparin-binding domain. While the role of VEGF is crucial to normal tissue function by inducing endothelial proliferation and vascular permeability, VEGF can also be produced by tumor cells and tumor-associated stromal cells, promoting tumor growth and metastases [30-32]. 


\section{The role of HIF transcription factors in NSCLC}

Protein levels of HIF1a and HIF2a are frequently over-expressed in NSCLC. In a study of 108 patients with early stage resectable NSCLC, HIF1a and HIF2a protein expression by immunohistochemisty (IHC) was found in 62 and $50 \%$ of samples, respectively [33]. Despite the fact that more samples appeared to have high levels of HIF1a expression, only elevated HIF2a expression correlated with an increased density of microvessels that stained positively for the VEGF--VEGFR-2 complex [33]. In a separate study of 74 early NSCLC stage patients, high levels of HIF1a expression by IHC correlated with a decreased disease free survival as well as carbonic anhydrase IX (CA IX) staining. Only CA IX expression, however, was found to be an independent prognostic factor by multivariate analysis [34].

The interaction between HIF2a and NSCLC has been examined genetically in a KRasinduced mouse model of lung cancer that dually expresses stabilized HIF2 $a$ and mutant $K R a s{ }^{G 12 D}$ [35]. Lung tumors from these mice were larger and resulted in a decreased overall survival when compared with mice with mutant KRas expression alone.

Additionally, production of stabilized HIF2a led to the development of tumors with a poorly differentiated, pleomorphic histology that displayed markers of epithelial to mesenchymal transition (EMT). Consistent with HIF's role in promoting angiogenesis, the HIF2aproducing lung tumors were found to have increased vascularity and blood flow associated with higher levels of angiogenic, bone-marrow-derived circulating endothelial progenitor cells (CEPs) in their peripheral blood. This finding suggests that tumors that produce HIF2a can mobilize CEPs from the bone marrow. Collectively, these results build upon the clinical data implicating HIF2 $a$ as a poor prognostic factor in human NSCLC and demonstrate in an autochthonous cancer model that HIF2a can promote lung tumor progression [35].

\section{Current state of anti-angiogenic therapy for NSCLC}

In vitro studies have shown that the levels of hypoxia-induced VEGF mRNA in highly metastatic lung cancer cells are significantly greater than those for lung cancer cell lines with low metastatic potential [36]. Similarly, in human NSCLC, high $V E G F$ mRNA levels have been correlated with shorter survival and earlier post-operative relapse rates [37]. The molecular evidence that VEGF plays an important role in the development of aberrant tumor vasculature in NSCLC has resulted in the development of targeted therapies that prevent unregulated angiogenesis and promote tumor regression.

\subsection{VEGF inhibition: bevacizumab}

Bevacizumab is a humanized monoclonal antibody with a high affinity to soluble VEGF. By binding VEGF in the circulation, bevacizumab decreases the likelihood of VEGF engaging its receptor. This strategy for inhibiting the VEGF axis has been shown to be clinically useful in a number of solid tumors including NSCLC (Table 1). An early randomized Phase II trial that enrolled 99 patients with advanced-stage NSCLC assigned patients to receive doublet chemotherapy with carboplatin and paclitaxel with or without bevacizumab dosed at either 7.5 or $15 \mathrm{mg} / \mathrm{kg}$ [38]. The median time to progression (TTP) was significantly longer in patients who received chemotherapy with the higher dose of bevacizumab relative to patients who received chemotherapy only or patients who received chemotherapy with the lower dose of bevacizumab.

These encouraging results led to the pivotal randomized Phase III trial, Eastern Cooperative Oncology Group (ECOG) 4599, in which 878 patients with Stage IIIB or IV NSCLC were randomized to receive carboplatin and paclitaxel with or without bevacizumab at $15 \mathrm{mg} / \mathrm{kg}$ every three weeks [3]. Based on the toxicities seen in the randomized Phase II trial above, patients with hemoptysis, squamous histology and CNS metastases were excluded. Patients 
receiving carboplatin/paclitaxel with bevacizumab had a statistically significant longer median overall survival than patients receiving carboplatin/paclitaxel with placebo (12.3 months and 10.3 months respectively). A second Phase III trial (Avastin in Lung Study (AVAiL)) comparing the combination of cisplatin and gemcitabine with or without bevacizumab in advanced-stage NSCLC patients confirmed an improvement in progressionfree survival (PFS)and objective response rates in patients receiving chemotherapy plus bevacizumab compared with those receiving chemotherapy alone [39]. It is currently not known whether the addition of bevacizumab to adjuvant chemotherapy regimens improves clinical outcomes. An ongoing cooperative group trial, ECOG 1505, hopes to address this question in patients with surgically resected stage IB, II, and III NSCLC.

\subsection{VEGF-R tyrosine kinase inhibitors}

Bevacizumab directly targets the VEGF ligand; however, small-molecule tyrosine kinase inhibitors (TKIs), which bind to and inhibit the interaction of VEGFR with ATP, have also shown promise in treating NSCLC. Sorafenib is a multi-targeted small molecule TKI that blocks several kinases including the RAF kinase, VEGFR-2, VEGFR-3, C-KIT, and platelet-derived growth factor receptor (PDGFR), as well as FMS proto-oncogene-like tyrosine kinase 3 (FLT3) and receptor tyrosine kinase proto-oncogene (RET) [40]. In a Phase II single-arm study of 52 patients with advanced NSCLC of all histologies, an overall response rate of $29 \%$ was seen in patients receiving single-agent sorafenib at the standard dose of $400 \mathrm{mg}$ twice daily [41]. Based on the bevacizumab trials, which suggested increased efficacy when combined with chemotherapy, a randomized Phase III trial (Evaluation of Sorafenib, Carboplatin, and Paclitaxel Efficacy in NSCLC (ESCAPE)) was performed that compared carboplatin/paclitaxel with or without sorafenib in 926 chemotherapy-naïve patients with advanced NSCLC [42]. This trial was closed early after a planned interim analysis revealed no difference in response rate, progression free survival or overall survival between the two groups. A subset analysis showed poorer overall survival (OS) in patients with squamous histology; therefore, these patients were subsequently excluded from the ongoing Phase III NSCLC research Experience Utilizing Sorafenib (NExuS) trial that is evaluating cisplatin and gemcitabine with or without sorafenib.

Sunitinib is a small-molecule tyrosine kinase inhibitor of VEGFR, PDGFR, C-KIT, and FLT-3 that has been examined for clinical activity in NSCLC. A randomized Phase II trial of 63 patients with platinum-refractory, advanced NSCLC received single agent sunitinib at the standard dose (50mg/day for four weeks on treatment, two weeks off treatment) [43]. An overall response rate of $11 \%$ was seen, and an additional $29 \%$ of patients had stable disease with a median survival of 23.9 weeks. A second Phase II trial examining the efficacy of continuously dosed sunitinib at $37.5 \mathrm{mg}$ in patients with refractory NSCLC showed a low overall response rate of only $2 \%$ but a one-year survival of $38 \%$ [44]. A randomized, double-blind, placebo controlled, Phase III cooperative group trial, Cancer and Leukemia Group B (CALGB) 30607, of sunitinib as maintenance therapy in non-progressing patients following an initial four cycles of platinum-based chemotherapy is currently ongoing [45].

\subsection{Dual VEGF and EGFR blockade}

Inhibition of EGFR in NSCLC is often cited as an example of therapeutic success in targeted therapy [46]. The prevalence of activating EGFR mutations in NSCLC is approximately 10 and $40 \%$ in North America/Western Europe and East Asia respectively [46]. These patients are typically female with an increased likelihood of being never-smokers. While the majority of EGFR mutations are either in-frame deletions of exon 19 or single-amino acid substitutions of exon 21, approximately $10 \%$ involve exon 18 or exon 20, the latter of which can confer resistance to EGFR TKI therapy [46]. It is now clear that EGFR mutations confer sensitivity to EGFR inhibition and that treatment of patients with advanced NSCLC 
harboring EGFR mutations with the small-molecule EGFR inhibitor, gefitinib, can result in a significant prolongation in PFS [47-49].

As activation of EGFR has been shown to upregulate VEGF levels, it is postulated that EGFR dependent increases in VEGF levels may result in decreased efficacy of agents targeting EGFR [50]. In this regard, dual inhibition of both pathways may provide a mechanism for overcoming this mode of resistance. Vandetanib, a small molecule TKI of both VEGFR and EGFR (as well as RET kinase) has been studied in four Phase II trials as either a single agent or in combination with chemotherapy [51-54]. These trials in both chemotherapy naïve and previously treated patients have shown encouraging results with notable improvements in PFS compared with standard chemotherapy regimens alone or the EGFR inhibitor gefitinib. Whether dual VEGFR/EGFR inhibition with vandetanib as a single agent or in combination with chemotherapy truly has clinical benefit is being tested in four randomized, double blind, placebo-controlled Phase III trials (Zactima Efficacy when Studied versus Tarceva (ZEST), Zactima in Combination with Docetaxel In non-small cell lung Cancer (ZODIAC), Zactima Efficacy with Alimta in Lung cancer (ZEAL), and Zactima Efficacy trial for NSCLC Patients with History of EGFR and Chemo-Resistance (ZEPHYR)).

An alternative approach to dual EGFR/VEGFR targeting is the combined use of bevacizumab and erlotinib. After a Phase I/II trial of patients with advanced stage NSCLC showed no dose-limiting toxicities, a randomized, double-blind, placebo-controlled Phase III study (ATLAS) enrolled 1157 patients (who were non-progressing after four cycles of platinum based chemotherapy plus bevacizumab) to receive maintenance bevacizumab every three weeks with or without erlotinib [55]. The data safety monitoring committee (DSMC) recommended halting the trial early after a pre-planned interim analysis demonstrated a significant improvement in progression free survival (the primary endpoint of the study). A second randomized Phase III trial (the Bevacizumab/Tarceva (BeTa) lung trial) evaluated the addition of erlotinib to bevacizumab as second-line therapy in patients with advanced NSCLC [56]. Although the primary endpoint of improved overall survival was not met, PFS was doubled in patients enrolled on the combination arm relative to those treated with erlotinib alone (3.4 months versus 1.7 months). In aggregate, these data suggest a benefit, in select NSCLC populations, to the combined inhibition of the VEGFR and EGFR compared with either alone.

\section{Potential biomarkers to predict response to anti-angiogenic therapy}

Finding an accurate and consistent biomarker to predict response to targeted anti-angiogenic therapies has been a challenge. This is at least partially due to the complexities and redundancies found within angiogenic pathways as well as the cytostatic effects (as opposed to the cytotoxic effects of chemotherapy) of molecular treatments. Therefore, there is a need to develop markers that can predict the long-term efficacy of these agents early in the treatment course, particularly in light of their cost and toxicity.

\subsection{Plasma or serum VEGF}

While many markers of angiogenesis in NSCLC have been investigated, the ability of cytokines and angiogenic growth factors in the serum or tumor to accurately predict response or prognosis has been variable. A 2006 review of prognostic markers of angiogenesis in NSCLC found that 10 of 16 studies reported elevated serum VEGF levels as being associated with a poor prognosis [57]. At least partially in keeping with this notion, a prospective correlative study of ECOG 4599 analyzed the relationship between baseline plasma VEGF levels and clinical outcomes. In this study, baseline VEGF levels appeared to be predictive of response to bevacizumab treatment. For patients with low levels of VEGF, 
individuals had the same response rate regardless of whether they were treated with chemotherapy alone or chemotherapy plus bevacizumab. In contrast, patients with high levels of VEGF were more likely to respond to bevacizumab, with a $33 \%$ overall response rate in patients receiving chemotherapy plus bevacizumab versus a $7 \%$ response rate in patients receiving chemotherapy alone [58]. While there was no association between baseline VEGF levels and overall survival, patients with low baseline serum VEGF had a statistically significant prolonged disease-free survival ( 6 months versus 4.5 months) regardless of treatment.

Hanrahan et al. retrospectively examined the predictive role of baseline plasma VEGF levels in patients receiving vandetanib in three separate randomized Phase II trials [59]. In a study of 168 patients randomized to receive vandetanib versus gefitinib, patients receiving vandetanib with low VEGF levels at baseline had superior PFS compared with patients receiving gefitinib. In a separate trial in which patients were treated with vandetanib combined with docetaxel versus docetaxel with placebo, patients with low baseline VEGF levels that received a lower dose of vandetanib combined with docetaxel had longer PFS compared with those treated with doctaxel plus placebo [53]. There was no difference in survival between the two treatment groups in patients with high VEGF levels. While the molecular basis behind these correlations remains unknown, potential explanations include the inability of vandetanib to fully achieve adequate VEGFR blockade in the face of higher VEGF levels. Alternatively, increased VEGF levels may merely be a surrogate marker for alternate, VEGF-independent modes of angiogenesis.

\subsection{Cytokines and angiogenic factors (CAFs)}

Increases in serum or plasma VEGF and decreases in soluble VEGFR 2 (sVEGFR-2) in response to VEGFR inhibition have been reported [60]. Along these lines, Hanrahan and colleagues used cytokine arrays to analyze the profiles of 35 cytokines and angiogenic factors (CAFs) and correlated the changes in their levels with the risk of progression [61]. Plasma from 123 NSCLC patients enrolled in a randomized Phase II trial of vandetanib monotherapy, carboplatin and paclitaxel $(\mathrm{CP})$, or the combination of vandetanib and chemotherapy (VCP), was analyzed at distinct time points during treatment [61]. In the vandetanib-only arm, VEGF levels had significantly increased by day 43 of treatment while sVEGFR-2 levels decreased in keeping with reports from other trials involving VEGFR TKIs [60]. There were also distinct patterns of CAF expression changes in chemotherapy treated patients, regardless of whether they received vandetanib. These included decreased levels of IL-12, IL-1 receptor antagonist (IL-1 RA) and MMP-9 [61]. Because white blood cells are a common source of these factors, the authors postulated that this decrease could be a result of treatment effects on leukocytes.

In each treatment arm, changes in the levels of different CAFs were associated with the risk of disease progression [61]. For example, patients receiving VCP had increases in IL-8, which interestingly has been found to be a compensatory mechanism of inducing VEGF expression and angiogenesis in colon cancer and renal cell carcinoma [62,63]. In patients receiving chemotherapy alone, increases in MMP-9 were associated with increased risk of progression [61]. This proangiogenic factor, which is expressed by tumor cells or delivered to the tumor microenvironment by pro-angiogenic bone-marrow-derived cells (BMDCs), plays a crucial role in development of tumor neovasculature [64]. Finally, in patients receiving vandentanib monotherapy as well as those treated with $\mathrm{VCP}$, there was a significantly longer PFS in patients with increased levels of intracellular cell adhesion molecule-1 (ICAM-1) on day 8 of treatment. Similar findings were seen in the plasma from bevacizumab-treated patients on ECOG 4599 in which baseline ICAM-1 levels were prognostic for survival and predictive of response to chemotherapy with or without bevacizumab [58]. 


\subsection{Intratumoral VEGF}

While measuring plasma levels of VEGF is appealing due to its convenience and accessibility, VEGF tumor expression has also been investigated as a biomarker of prognosis $[65,66]$. The expression of four VEGF mRNA isoforms was examined in 57 NSCLC tumors by reverse transcriptase-PCR (RT-PCR) and correlated with markers of angiogenesis, patient survival and postoperative relapse. A high level of $\mathrm{VEGF}_{189} \mathrm{mRNA}$ was associated with a high microvessel count within the tumor as well as a shorter survival and earlier postoperative relapse [37]. Other studies have shown, however, that no clear association exists between the amount of VEGF detected in tumors with that of circulating plasma levels [67-69]. From the above data, it remains clear that more precise and predictive biomarkers of response to VEGF-targeted agents are necessary.

\section{Expert opinion}

Inhibition of the VEGF axis has proven to be a tractable therapeutic strategy in the treatment of NSCLC, yet ultimately patients' tumors progress despite these therapies. A better understanding of the molecular or microenvironmental events and conditions that lead to VEGF upregulation will result in improved treatments for NSCLC and a more effective selection of patients most likely to respond to anti-angiogenic therapy. Intratumoral hypoxia is found relatively frequently in NSCLC and is a well known regulator of VEGF levels. There are a number of other molecular alterations that lead to VEGF upregulation in NSCLC such as activation of the MEK/ERK and PI3K pathways via activating mutations of KRAS, EGFR and PI3K or loss of phosphatase and tensin homologue (PTEN) (Figure 2) $[15,16,70,71]$. While MEK/ERK and PI3K activation have been shown to elevate HIF mRNA levels, activation of these pathways likely increases VEGF levels in both HIFdependent and -independent manners.

A second common path to VEGF upregulation in NSCLC is activation of the mTOR pathway. Activation of mTOR is known to increase HIF and VEGF levels both transcriptionally and translationally, and in NSCLC mTOR can be activated by a number of genetic events such as loss of the tuberous sclerosis complex, PTEN, or LKB1 tumor suppressor genes (Figure 2) [72]. While homozygous deletion of the tuberous sclerosis complex (either TSC1 or TSC2) appears to be a relatively rare event in NSCLC, somatic mutations of the PTEN and LKB1 tumor suppressor genes have been found in up to 6 and $30 \%$ of NSCLC patients respectively [73-75]. Because inactivation of tumor suppressor genes such as TSC1/2, PTEN and LKB1 are loss of function mutations, these genetic events have typically proven to be difficult therapeutic targets. However, targeting mTOR may result in the silencing of the downstream effects of their loss. This therapeutic strategy is being actively investigated in clinical trials aimed at NSCLC.

The past decade has delivered a clearer understanding of the genetic events involved in the development and progression of NSCLC, including critical mutations that are targetable, such as EGFR, and those that remain elusive therapeutic targets, such as KRAS. Moreover, clinical studies have confirmed the importance of the VEGF pathway and other angiogenic molecules as modulators of the tumor microenvironment. Recent studies using genetically engineered mouse models expressing stabilized forms of HIF point towards the importance of HIF in the evolution of NSCLC angiogenesis and tumor progression and demonstrate genetically that HIF is causally involved in NSCLC. These insights as well as the defined role of VEGF axis inhibitors in the treatment of NSCLC will impart clues as to the modes of resistance to angiogenesis inhibitors and further possible targeting strategies. 


\section{Acknowledgments}

WY Kim is funded by the Uniting against Lung Cancer Foundation, Damon Runyon Foundation, NIH/NCI.

\section{Bibliography}

1. Jemal A, Siegel R, Ward E, et al. Cancer statistics, 2009. CA Cancer J Clin. 2009; 59:225-49. [PubMed: 19474385]

2. Schiller JH, Harrington D, Belani CP, et al. Comparison of four chemotherapy regimens for advanced non-small-cell lung cancer. N Engl J Med. 2002; 346:92-8. [PubMed: 11784875]

3. Sandler A, Gray R, Perry MC, et al. Paclitaxel-carboplatin alone or with bevacizumab for nonsmall-cell lung cancer. N Engl J Med. 2006; 355:2542-50. [PubMed: 17167137]

4. Folkman J. What is the evidence that tumors are angiogenesis dependent? J Natl Cancer Inst. 1990; 82:4-6. [PubMed: 1688381]

5. Bergers G, Benjamin LE. Tumorigenesis and the angiogenic switch. Nat Rev Cancer. 2003; 3:40110. [PubMed: 12778130]

6. Folkman J. Tumor angiogenesis: therapeutic implications. N Engl J Med. 1971; 285:1182-6. [PubMed: 4938153]

7. Hanahan D, Folkman J. Patterns and emerging mechanisms of the angiogenic switch during tumorigenesis. Cell. 1996; 86:353-64. [PubMed: 8756718]

8. Hirschi KK, D’Amore PA. Pericytes in the microvasculature. Cardiovasc Res. 1996; 32:687-98. [PubMed: 8915187]

9. Jain RK. Normalization of tumor vasculature: an emerging concept in antiangiogenic therapy. Science. 2005; 307:58-62. [PubMed: 15637262]

10. Folberg R, Hendrix MJ, Maniotis AJ. Vasculogenic mimicry and tumor angiogenesis. Am J Pathol. 2000; 156:361-81. [PubMed: 10666364]

11. McDonald DM, Munn L, Jain RK. Vasculogenic mimicry: how convincing, how novel, and how significant? Am J Pathol. 2000; 156:383-8. [PubMed: 10666365]

12. Fukumura D, Jain RK. Imaging angiogenesis and the microenvironment. APMIS. 2008; 116:695715. [PubMed: 18834413]

13. Semenza GL. Targeting HIF-1 for cancer therapy. Nat Rev Cancer. 2003; 3:721-32. [PubMed: 13130303]

14. Kim WY, Kaelin WG. Role of VHL gene mutation in human cancer. J Clin Oncol. 2004; 22:49915004. [PubMed: 15611513]

15. Sodhi A, Montaner S, Miyazaki H, Gutkind JS. MAPK and Akt act cooperatively but independently on hypoxia inducible factor-1alpha in rasV12 upregulation of VEGF. Biochem Biophys Res Commun. 2001; 287:292-300. [PubMed: 11549290]

16. Blancher C, Moore JW, Robertson N, Harris AL. Effects of ras and von Hippel-Lindau (VHL) gene mutations on hypoxia-inducible factor (HIF)-1alpha, HIF-2alpha, and vascular endothelial growth factor expression and their regulation by the phosphatidylinositol $3^{\prime}$-kinase/Akt signaling pathway. Cancer Res. 2001; 61:7349-55. [PubMed: 11585776]

17. Kumar SM, Yu H, Edwards R, et al. Mutant V600E BRAF increases hypoxia inducible factor-1alpha expression in melanoma. Cancer Res. 2007; 67:3177-84. [PubMed: 17409425]

18. Hudson CC, Liu M, Chiang GG, et al. Regulation of hypoxia-inducible factor 1alpha expression and function by the mammalian target of rapamycin. Mol Cell Biol. 2002; 22:7004-14. [PubMed: 12242281]

19. Thomas GV, Tran C, Mellinghoff IK, et al. Hypoxia-inducible factor determines sensitivity to inhibitors of mTOR in kidney cancer. Nat Med. 2006; 12:122-7. [PubMed: 16341243]

20. Treins C, Giorgetti-Peraldi S, Murdaca J, et al. Insulin stimulates hypoxia-inducible factor 1 through a phosphatidylinositol 3-kinase/target of rapamycin-dependent signaling pathway. J Biol Chem. 2002; 277:27975-81. [PubMed: 12032158]

21. Iyer NV, Kotch LE, Agani F, et al. Cellular and developmental control of O2 homeostasis by hypoxia-inducible factor 1alpha. Genes Dev. 1998; 12:149-62. [PubMed: 9436976] 
22. Compernolle V, Brusselmans K, Acker T, et al. Loss of HIF-2alpha and inhibition of VEGF impair fetal lung maturation, whereas treatment with VEGF prevents fatal respiratory distress in premature mice. Nat Med. 2002; 8:702-10. [PubMed: 12053176]

23. Tian H, Hammer RE, Matsumoto AM, et al. The hypoxia-responsive transcription factor EPAS1 is essential for catecholamine homeostasis and protection against heart failure during embryonic development. Genes Dev. 1998; 12:3320-4. [PubMed: 9808618]

24. Hu CJ, Sataur A, Wang L, et al. The N-terminal transactivation domain confers target gene specificity of hypoxia-inducible factors HIF-1alpha and HIF-2alpha. Mol Biol Cell. 2007; 18:4528-42. [PubMed: 17804822]

25. Hu CJ, Wang LY, Chodosh LA, et al. Differential roles of hypoxia-inducible factor 1alpha (HIF-1alpha) and HIF-2alpha in hypoxic gene regulation. Mol Cell Biol. 2003; 23:9361-74. [PubMed: 14645546]

26. Kim WY, Safran M, Buckley RM, et al. Failure to prolyl hydroxylate HIFalpha phenocopies VHL inactivation in vivo. EMBO J. 2006; 25:4650-62. [PubMed: 16977322]

27. Raval RR, Lau KW, Tran MG, et al. Contrasting properties of hypoxia-inducible factor 1 (HIF-1) and HIF-2 in von Hippel-Lindau-associated renal cell carcinoma. Mol Cell Biol. 2005; 25:567586. [PubMed: 15964822]

28. Wang V, Davis DA, Haque M, et al. Differential gene up-regulation by hypoxia-inducible factor-1alpha and hypoxia-inducible factor-2alpha in HEK293T cells. Cancer Res. 2005; 65:3299_ 306. [PubMed: 15833863]

29. Ellis LM, Hicklin DJ. VEGF-targeted therapy: mechanisms of anti-tumour activity. Nat Rev Cancer. 2008; 8:579-91. [PubMed: 18596824]

30. Donnem T, Al-Shibli K, Al-Saad S, et al. VEGF-A and VEGFR-3 correlate with nodal status in operable non-small cell lung cancer: inverse correlation between expression in tumor and stromal cells. Lung Cancer. 2009; 63:277-83. [PubMed: 18599153]

31. Lewis JS, Landers RJ, Underwood JC, et al. Expression of vascular endothelial growth factor by macrophages is up-regulated in poorly vascularized areas of breast carcinomas. J Pathol. 2000; 192:150-8. [PubMed: 11004690]

32. Pinto MP, Badtke MM, Dudevoir ML, et al. Vascular endothelial growth factor secreted by activated stroma enhances angiogenesis and hormone-independent growth of estrogen receptorpositive breast cancer. Cancer Res. 2010; 70:2655-64. [PubMed: 20332242]

33. Giatromanolaki A, Koukourakis MI, Sivridis E, et al. Relation of hypoxia inducible factor 1alpha and 2alpha in operable non-small cell lung cancer to angiogenic/molecular profile of tumours and survival. Br J Cancer. 2001; 85:881-90. [PubMed: 11556841]

34. Kim SJ, Rabbani ZN, Dewhirst MW, et al. Expression of HIF-1alpha, CA IX, VEGF, and MMP-9 in surgically resected non-small cell lung cancer. Lung Cancer. 2005; 49:325-35. [PubMed: 15935515]

35. Kim WY, Perera S, Zhou B, et al. HIF2alpha cooperates with RAS to promote lung tumorigenesis in mice. J Clin Invest. 2009; 119:2160-70. [PubMed: 19662677]

36. Koshikawa N, Iyozumi A, Gassmann M, Takenaga K. Constitutive upregulation of hypoxiainducible factor-1alpha mRNA occurring in highly metastatic lung carcinoma cells leads to vascular endothelial growth factor overexpression upon hypoxic exposure. Oncogene. 2003; 22:6717-24. [PubMed: 14555985]

37. Yuan A, Yu CJ, Kuo SH, et al. Vascular endothelial growth factor 189 mRNA isoform expression specifically correlates with tumor angiogenesis, patient survival, and postoperative relapse in nonsmall-cell lung cancer. J Clin Oncol. 2001; 19:432-41. [PubMed: 11208836]

38. Johnson DH, Fehrenbacher L, Novotny WF, et al. Randomized Phase II trial comparing bevacizumab plus carboplatin and paclitaxel with carboplatin and paclitaxel alone in previously untreated locally advanced or metastatic non-small-cell lung cancer. J Clin Oncol. 2004; 22:218491. [PubMed: 15169807]

39. Reck M, von Pawel J, Zatloukal P, et al. Phase III trial of cisplatin plus gemcitabine with either placebo or bevacizumab as first-line therapy for nonsquamous non-small-cell lung cancer: AVAil. J Clin Oncol. 2009; 27:1227-34. [PubMed: 19188680] 
40. Gridelli C, Maione P, Del Gaizo F, et al. Sorafenib and sunitinib in the treatment of advanced nonsmall cell lung cancer. Oncologist. 2007; 12:191-200. [PubMed: 17296815]

41. Blumenschein GR Jr, Gatzemeier U, Fossella F, et al. Phase II, multicenter, uncontrolled trial of single-agent sorafenib in patients with relapsed or refractory, advanced non-small-cell lung cancer. J Clin Oncol. 2009; 27:4274-80. [PubMed: 19652055]

42. Scagliotti G, Novello S, von Pawel J, et al. Phase III study of carboplatin and paclitaxel alone or with sorafenib in advanced non-small-cell lung cancer. J Clin Oncol. 2010; 28:1835-42. [PubMed: 20212250]

43. Socinski MA, Novello S, Brahmer JR, et al. Multicenter, Phase II trial of sunitinib in previously treated, advanced non-small-cell lung cancer. J Clin Oncol. 2008; 26:650-6. [PubMed: 18235126]

44. Novello S, Scagliotti GV, Rosell R, et al. Phase II study of continuous daily sunitinib dosing in patients with previously treated advanced non-small cell lung cancer. Br J Cancer. 2009; 101:1543-8. [PubMed: 19826424]

45. [Last accessed 28 July 2010] NCT00693992. Randomized, Phase III, Double-Blind PlaceboControlled Trial of Sunitinib (NSC \#736511, IND \#74019) as Maintenance Therapy in NonProgressing Patients Following an Initial Four Cycles of Platinum-Based Combination Chemotherapy in Advanced, Stage IIIB / IV Non-Small Cell Lung Cancer. 2010. Available from: http://clinicaltrials.gov/ct2/show/NCT00693992?term=NCT00693992\&rank=1

46. Sharma SV, Bell DW, Settleman J, Haber DA. Epidermal growth factor receptor mutations in lung cancer. Nat Rev Cancer. 2007; 7:169-81. [PubMed: 17318210]

47. Paez JG, Janne PA, Lee JC, et al. EGFR mutations in lung cancer: correlation with clinical response to gefitinib therapy. Science. 2004; 304:1497-500. [PubMed: 15118125]

48. Lynch TJ, Bell DW, Sordella R, et al. Activating mutations in the epidermal growth factor receptor underlying responsiveness of non-small-cell lung cancer to gefitinib. N Engl J Med. 2004; 350:2129-39. [PubMed: 15118073]

49. Maemondo M, Inoue A, Kobayashi K, et al. Gefitinib or chemotherapy for non-small-cell lung cancer with mutated EGFR. N Engl J Med. 2010; 362:2380-8. [PubMed: 20573926]

50. Camp ER, Summy J, Bauer TW, et al. Molecular mechanisms of resistance to therapies targeting the epidermal growth factor receptor. Clin Cancer Res. 2005; 11:397-405. [PubMed: 15671571]

51. Heymach JV, Johnson BE, Prager D, et al. Randomized, placebo-controlled Phase II study of vandetanib plus docetaxel in previously treated non small-cell lung cancer. J Clin Oncol. 2007; 25:4270-7. [PubMed: 17878479]

52. Heymach JV, Paz-Ares L, De Braud F, et al. Randomized Phase II study of vandetanib alone or with paclitaxel and carboplatin as first-line treatment for advanced non-small-cell lung cancer. J Clin Oncol. 2008; 26:5407-15. [PubMed: 18936474]

53. Kiura K, Nakagawa K, Shinkai T, et al. A randomized, double-blind, Phase IIa dose-finding study of Vandetanib (ZD6474) in Japanese patients with non-small cell lung cancer. J Thorac Oncol. 2008; 3:386-93. [PubMed: 18379357]

54. Natale RB, Bodkin D, Govindan R, et al. Vandetanib versus gefitinib in patients with advanced non-small-cell lung cancer: results from a two-part, double-blind, randomized Phase II study. J Clin Oncol. 2009; 27:2523-9. [PubMed: 19332730]

55. Miller VA, O'Connor P, Soh C, Kabbinavar F. A randomized, double-blind, placebo-controlled, phase IIIb trial (ATLAS) comparing bevacizumab (B) therapy with or without erlotinib (E) after completion of chemotherapy with B for first-line treatment of locally advanced, recurrent, or metastatic non-small cell lung cancer (NSCLC). Annual Meeting Proceedings 2009. J Clin Oncol. 2009; 27(18S):LBA8002.

56. Herbst, RS.; Stern, H.; Amler, L., et al. Biomarker evaluation in the Phase III, placebo (P)controlled, randomized beta trial of bevacizumab (B) and erlotinib (E) for patients (Pts) with advanced Non-Small Cell Lung Cancer (NSCLC) after failure of standard 1st-line chemotherapy: correlation with treatment outcomes. Proceedings of the International Association for the Study of Lung Cancer 2009; San Francisco, CA. 2009. p. 81

57. Delmotte P, Martin B, Paesmans M, et al. VEGF and survival of patients with lung cancer: a systematic literature review and meta-analysis. Rev Mal Respir. 2002; 19:577-84. [PubMed: 12473944] 
58. Dowlati A, Gray R, Sandler AB, et al. Cell adhesion molecules, vascular endothelial growth factor, and basic fibroblast growth factor in patients with non-small cell lung cancer treated with chemotherapy with or without bevacizumab - an Eastern Cooperative Oncology Group Study. Clin Cancer Res. 2008; 14:1407-12. [PubMed: 18316562]

59. Hanrahan EO, Ryan AJ, Mann H, et al. Baseline vascular endothelial growth factor concentration as a potential predictive marker of benefit from vandetanib in non-small cell lung cancer. Clin Cancer Res. 2009; 15:3600-9. [PubMed: 19447868]

60. Norden-Zfoni A, Desai J, Manola J, et al. Blood-based biomarkers of SU11248 activity and clinical outcome in patients with metastatic imatinib-resistant gastrointestinal stromal tumor. Clin Cancer Res. 2007; 13:2643-50. [PubMed: 17473195]

61. Hanrahan EO, Lin HY, Kim ES, et al. Distinct patterns of cytokine and angiogenic factor modulation and markers of benefit for vandetanib and/or chemotherapy in patients with non-smallcell lung cancer. J Clin Oncol. 2010; 28:193-201. [PubMed: 19949019]

62. Mizukami Y, Jo WS, Duerr EM, et al. Induction of interleukin-8 preserves the angiogenic response in HIF-1alpha-deficient colon cancer cells. Nat Med. 2005; 11:992-7. [PubMed: 16127434]

63. Huang D, Ding Y, Zhou M, et al. Interleukin-8 mediates resistance to antiangiogenic agent sunitinib in renal cell carcinoma. Cancer Res. 2010; 70:1063-71. [PubMed: 20103651]

64. Du R, Lu KV, Petritsch C, et al. HIF1alpha induces the recruitment of bone marrow-derived vascular modulatory cells to regulate tumor angiogenesis and invasion. Cancer Cell. 2008; 13:206-20. [PubMed: 18328425]

65. Donnem T, Al-Saad S, Al-Shibli K, et al. Co-expression of PDGF-B and VEGFR-3 strongly correlates with lymph node metastasis and poor survival in non-small-cell lung cancer. Ann Oncol. 2010; 21:223-31. [PubMed: 19628565]

66. Zhan P, Wang J, Lv XJ, et al. Prognostic value of vascular endothelial growth factor expression in patients with lung cancer: a systematic review with meta-analysis. J Thorac Oncol. 2009; 4:1094103. [PubMed: 19687765]

67. Fontanini G, Vignati S, Boldrini L, et al. Vascular endothelial growth factor is associated with neovascularization and influences progression of non-small cell lung carcinoma. Clin Cancer Res. 1997; 3:861-5. [PubMed: 9815760]

68. Imoto H, Osaki T, Taga S, et al. Vascular endothelial growth factor expression in non-small-cell lung cancer: prognostic significance in squamous cell carcinoma. J Thorac Cardiovasc Surg. 1998; 115:1007-14. [PubMed: 9605068]

69. Volm M, Koomagi R, Mattern J. Prognostic value of vascular endothelial growth factor and its receptor Flt-1 in squamous cell lung cancer. Int J Cancer. 1997; 74:64-8. [PubMed: 9036871]

70. Zhong H, Chiles K, Feldser D, et al. Modulation of hypoxia-inducible factor 1alpha expression by the epidermal growth factor/phosphatidylinositol 3-kinase/PTEN/AKT/FRAP pathway in human prostate cancer cells: implications for tumor angiogenesis and therapeutics. Cancer Res. 2000; 60:1541-5. [PubMed: 10749120]

71. Zundel W, Schindler C, Haas-Kogan D, et al. Loss of PTEN facilitates HIF-1-mediated gene expression. Genes Dev. 2000; 14:391-6. [PubMed: 10691731]

72. Hanna SC, Heathcote SA, Kim WY. mTOR pathway in renal cell carcinoma. Expert Rev Anticancer Ther. 2008; 8:283-92. [PubMed: 18279068]

73. Ji H, Ramsey MR, Hayes DN, et al. LKB1 modulates lung cancer differentiation and metastasis. Nature. 2007; 448:807-10. [PubMed: 17676035]

74. Liang MC, Ma J, Chen L, et al. TSC1 loss synergizes with KRAS activation in lung cancer development in the mouse and confers rapamycin sensitivity. Oncogene. 2010; 29:1588-97. [PubMed: 19966866]

75. Forbes S, Clements J, Dawson E, et al. COSMIC 2005. Br J Cancer. 2006; 94:318-22. [PubMed: 16421597]

76. Adjei AA, Mandrekar SJ, Dy GK, et al. Phase II trial of pemetrexed plus bevacizumab for secondline therapy of patients with advanced non-small-cell lung cancer: NCCTG and SWOG study N0426. J Clin Oncol. 2010; 28:614-19. [PubMed: 19841321]

77. Herbst RS, O'Neill VJ, Fehrenbacher L, et al. Phase II study of efficacy and safety of bevacizumab in combination with chemotherapy or erlotinib compared with chemotherapy alone for treatment 
of recurrent or refractory non small-cell lung cancer. J Clin Oncol. 2007; 25:4743-50. [PubMed: 17909199]

78. Lind JS, Dingemans AM, Groens HJ, et al. A multicenter, Phase II study of erlotinib and sorafenib in chemotherapy-naive patients with advanced non-small cell lung cancer. Clin Cancer Res. 2010; 16:3078-87. [PubMed: 20395213] 


\section{Article highlights}

- Several angiogenic molecules have been shown to play a key role in NSCLC angiogenesis including the VEGF and hypoxia-inducible factor families.

- Genetically engineered mouse models of lung cancer suggest that expression of HIF can promote more vascular, invasive and metastatic tumors.

- Current antiangiogenic strategies target VEGF through either humanized monoclonal antibodies (e.g., bevacizumab), by inhibition of the VEGF receptor by small-molecular tyrosine kinase inhibitors (e.g., sunitinib and sorafenib), or by dual VEGFR/EGFR inhibition (e.g., vandetanib).

- Bevacizumab in combination with chemotherapy has been shown to prolong overall survival in patients with advanced NSCLC.

- Several predictive biomarkers of response to anti-VEGF therapy are under evaluation including serum/plasma VEGF levels, cytokine and angiogenic growth factors, and intratumoral VEGF levels.

- There are multiple modes of VEGF upregulation, some of which are tenable therapeutic targets (e.g., PI3K or mTOR activation), others which are not (e.g., hypoxia). 


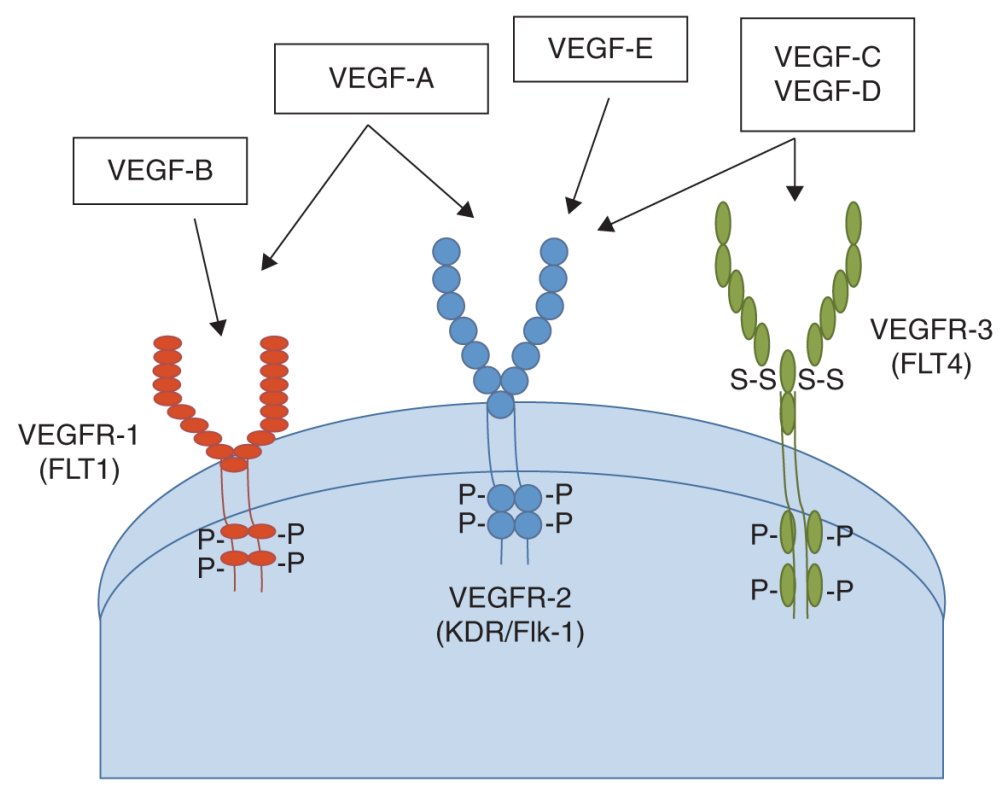

Figure 1. The VEGF receptors and ligands

VEGF comprises a family of endothelial growth factors that play a central role in angiogenesis through their effects on endothelial cell migration, proliferation, permeability and survival. VEGF-A is the most abundant of the ligands and binds to VEGFR-1 and VEGFR-2 (also known as FMS-like tyrosine kinase 1 (FLT1) and kinase insert domain protein receptor (KDR) fetal liver kinase-1 (Flk-1), respectively), the latter being considered a critical receptor in regulating angiogenesis. VEGFR-3 (also known as FLT4) is predominantly located on lymphatic endothelial cells and through binding with its ligands, VEGF-C and D, is thought to be primarily responsible for lymphagiogenesis. 


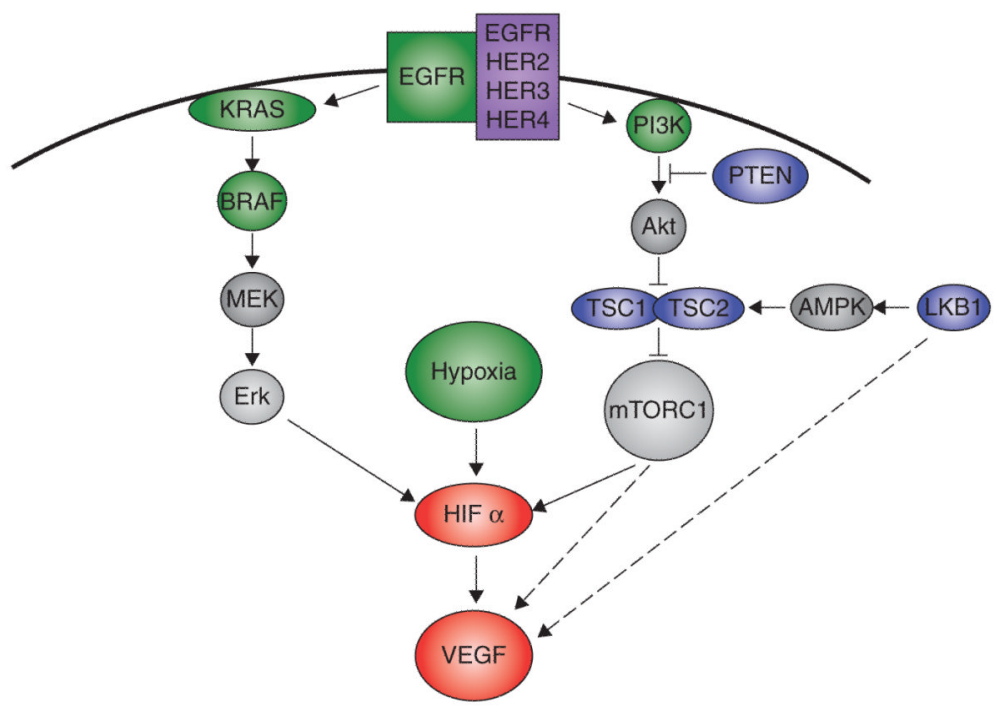

Figure 2. The molecular pathways leading to VEGF upregulation in NSCLC

Intratumoral hypoxia is found relatively frequently in NSCLC, and hypoxia --inducible factor (HIF) is a well-known regulator of VEGF levels. A number of other molecular alterations exist that probably lead to VEGF upregulation in NSCLC such as activation of the MEK/ERK and PI3K pathways via activating mutations of KRAS, EGFR and PI3K or loss of phosphatase and tensin homologue (PTEN). A second common route to VEGF upregulation in NSCLC is activation of the mammalian target of rapamycin (mTOR) pathway. Activation of mTOR is known to increase HIF and VEGF levels both transcriptionally and translationally, and in NSCLC mTOR can be activated by loss of the tuberous sclerosis complex, deletion of PTEN, or via inactivating mutations of the LKB1 tumor suppressor gene. Activating events are shown in green. Loss of function events in blue. AMPK: AMP-activated protein kinase. 


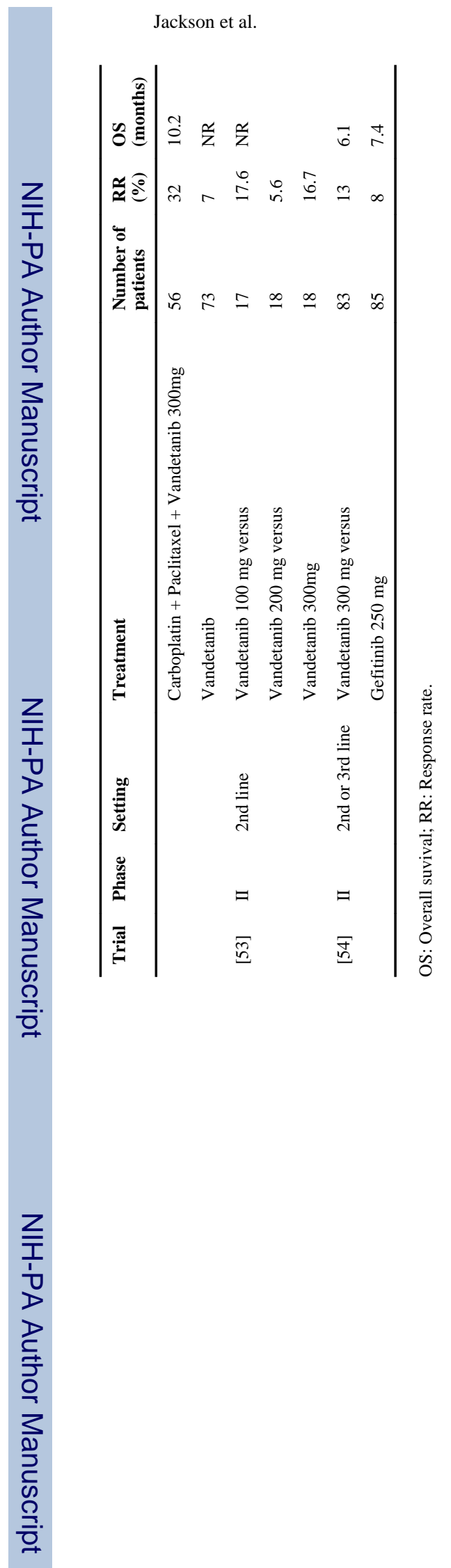

Expert Opin Ther Targets. Author manuscript; available in PMC 2013 May 07. 